\title{
Research on the Current Situation and Model of Commodity E-commerce Logistics in Ningbo
}

\author{
Qiying Shui ${ }^{1, a}$, Fei Ma ${ }^{2, b,{ }^{*},}$ Jiaqing Zhu ${ }^{3, c}$, \\ Jiaqi Qiu ${ }^{4, d}$, Suiyi Lin ${ }^{5, e}$ and Tianying Jiang ${ }^{6, f}$ \\ 1 6 Ningbo Dahongying University, Yinzhou District, Ningbo City, Zhejiang Province, China \\ a1596039797@qq.com, b1165490288@qq.com, c403775622@qq.com, \\ d1098896835@qq.com, e384535548@qq.com, fjty7608@126.com
}

Key words: commodities; electronic commerce; logistics ; mode.

Abstract. This paper analyzes the status of e-commerce logistics and commodity logistics model in Ningbo, and puts forward the problems of the logistics mode. At last, in order to solve the existing problems, it provides the corresponding countermeasures for the healthy and rapid development of the logistics mode.

\section{Introduction}

\subsection{Current situation of Commodity E-commerce in Ningbo}

E-commerce refers to different countries or regions of the parties to the transaction through the Internet and related information platform in the form of customs clearance, traditional trade show, negotiate and clinch a deal the link to implement the digital products import and export of new trade ${ }^{[9]}$ At present, the electronic commerce of Ningbo is developing continuously, and the Ningbo commodity electronic commerce market which takes the small and medium-sized enterprise as the main body also presents the good development situation ${ }^{[1]}$.

Tang Jun, deputy general manager of e-commerce Co., Ltd. reservoir in the storage and transportation of China Development Forum said: at present, commodity e-commerce development in the e-commerce transactions, from 2012 to 2015 in these three years, B2B transaction volume accounted for $80 \%$ of the total volume of transactions, and commodity trading volume accounted for the proportion of very large. It can be seen that the development of Commodity E-commerce has a decisive impact on the future economy of society ${ }^{[2]}$.

Ningbo rich natural resources, the implementation of the national "marine economy" development strategy, a national e-commerce pilot areas, e-commerce transactions in the National Engineering laboratory. The use of e-commerce platform, a new mode of commodity trade, investment, supply chain innovation of the Internet era, to achieve the "Internet plus goods + financial integration services, improve circulation efficiency and reduce the cost of circulation, boosting the traditional industries and the national economic transformation and upgrading.

\subsection{Current situation of Commodity E-commerce logistics in Ningbo}

1.2.1 Ningbo commerce logistics inadequate policy support

E-commerce in Ningbo started late, but the pace of development is amazing. Now the lack of electricity supplier logistics policy to support the building of Ningbo as a modern international port city. Compared with the developed areas, Ningbo's policy support is particularly inadequate. This prevents commodity e-commerce and the rapid development of logistics enterprises in a way.

1.2.2 Ningbo logistics infrastructure is not perfect

The time of logistics in Ningbo city is relatively late. Compared to other regions, there is a large gap in logistics environment. There are fewer transportation hubs connecting different modes of transport. All kinds of facilities also remains to be perfect. As a result of Commodity E-commerce logistics related to warehousing, distribution, transportation and a series of problems, we should to establish a efficient logistics system, and introduce more sophisticated and advanced logistics facilities. However, the current market of logistics and transport for a long time, many procedures, high cost of the phenomenon has violated the characteristics of e-commerce logistics fast and convenient. And which has seriously restricted the further development of Commodity E-commerce Logistics. 


\subsubsection{Lack of third party specialized logistics service}

Ningbo has a lot of third party logistics enterprises, But the number of the third party logistics enterprises with a high degree of specialization is not much. For logistics services, There is no special for Commodity E-commerce enterprises to provide a full range of professional logistics services. Specialized third party logistics service is necessary, which is conducive to the promotion of Ningbo commodity Electronics.

1.2.4 the speed of logistics development and the demand of electronic commerce is not coordinated Ningbo e-commerce development speed is very fast. The trading volume is about 1.6 Trillion yuan in 2011, about 2.0 Trillion yuan in 2012, about 3.1 yuan in 2013. The growth rate of its trade is relatively fast. Corresponding to the logistics enterprises, engaged in e-commerce is relatively small. Most are completed by the courier company logistics and distribution services. Such a large amount of logistics, relying solely on the courier company is far from enough, which gives the development of Commodity E-commerce logistics has brought a huge obstacle ${ }^{[3]}$.

\subsection{The present situation of Ningbo Commodity E-commerce logistics mode}

Commodity trading platform is the core of the whole process of e-commerce logistics model. Building set of electronic certification, online trading, online payment, online, online logistics supply chain financing, credit assessment, which integrates the entire online service system. With the transaction as the core, through the platform function and logistics business online collaboration, integrated trading platform internal and external logistics service system, the integration of social logistics resources, To achieve logistics and online certification, trading, payment, financing services seamlessly. Platform through the integration of information and business processes, integration of social resources, which provide customers with a fully integrated supply chain logistics service ${ }^{[4]}$.

\section{The problems of Commodity E-commerce logistics model in Ningbo}

\subsection{Information lag}

At present, There is a lag problem in all kinds of messages in Ningbo commodity electronic trading market. Basically, every logistics portal has a logistics tracking. But the logistics can not be tracked and updated in time. All the information is of great importance to the effectiveness and timeliness, So as to provide a guarantee for the value of information, so that "information" can be called information.

\subsection{Defects in logistics mode design}

The core of logistics model design is the trading platform and trading system ${ }^{[5]}$. Based on the online logistics and lack of offline field trip, the seller or the buyer can not fully grasp the initiative and control over logistics. It may increase transaction costs and disclose their trade secrets. At the same time, it is difficult to avoid the disadvantages, such as the investment is too large, inefficient, limited size, degree of specialization is not high.

\subsection{Defects in logistics management mode}

Receiving and delivery, be the link with logistics service providers to provide services, which has the biggest influence on the effect of the commodity electronic trading. The link has a lot of uncontrollable factors, it takes a long time, the risk is big, and the network degree is the worst. If the link is not good, will affect the entire transaction, like bringing losses to both sides and the logistics of the third parties, and even causing great harm to the online transactions ${ }^{[6]}$. Therefore, it is very important to monitor and track the network in real time. Logistics information transparency helps to smooth completion of the commodity electricity, implement end-to-end e-commerce.

\section{Ningbo bulk commodity e-commerce logistics model Development Strategy}

\subsection{Establish bulk commodity logistics innovation system}

To establish a more perfect logistics system, a clear innovation-driven development as the goal, forming a basic pattern. First of all, it focuses on the development of medium and long term electronic trading market, and the futures varieties of superior industrial clusters are selectively bred. Secondly, 
gradually build market logistics innovation model system, make it become a comprehensive professional trading, information, international trade and logistics center ${ }^{[7]}$. Finally, make full use of the port, trading market and other advantages, to build modern commodity trading markets innovative logistics model system.

\subsection{Develop bulk commodity logistics business model}

Adhere to optimize the logistics structure, vigorously develop and innovate the logistics business model, enhance the concentration of radiation, actively develop the electricity industry, and strive to build a logistics center. First of all, to build industrial clusters as the goal, make characteristics of the professional market become bigger and stronger. Secondly, accelerate the development of electrical business logistics public information platform, promote the logistics, finance, shipping and other industries into the informationization, digitization. In the end, the government of Ningbo should take an active role in bulk commodities e-commerce logistics informationization, to speed up the shift in the direction of intelligent and servitization ${ }^{[8]}$.

\subsection{Innovate bulk commodity logistics organization mode}

The development of logistics, the key is to enhance industrial policy innovation chain, financial services, logistics services, modern logistics organization mode. Firstly, give full play to the bulk commodity electricity suppliers have standardized processes, strong control and other obvious advantages, adjust the structure of the logistics industry. Secondly, the bulk commodity from static to dynamic reserves, increase the amount of existing reserves in Ningbo and save costs. Finally, build the market logistics organization mode, and speed up the formation of the price of the right to price trading base.

\section{Conclusion}

In recent years, Ningbo commodity markets e-commerce logistics policy support is insufficient, logistics development speed and e-commerce needs not harmonious, logistics infrastructure is not perfect, the lack of third-party logistics services. Caused the corresponding problems of e-commerce market logistics mode, logistics model validity and timeliness of information, logistics pattern design problems and logistics management model. And Ningbo commodity e-commerce logistics model want to be healthy and rapid development, cannot leave the Ningbo regional innovation system, build commodity logistics basic form innovation driven development pattern; Stick to optimize the structure of commodity e-commerce logistics, strive to build a commodity e-commerce logistics center; Increase in commodity industry organization mode of modern logistics, accelerate become important commodities trading base ${ }^{[10]}$.

\section{Acknowledgements}

This work was financially supported by the College Students of Science and Technology Innovation Plan and Planted Talent in Zhejiang Province in 2016, Commodity Logistics Industry Space-time Evolution and Influence Mechanism Research of Ningbo(Topic NO. 2016R425003) and Soft Science Project of Ningbo City in 2016, Research on Spatial Distribution Characteristics and Location Selection of Commodity Logistics Industry in Ningbo(Topic NO. 2016A10054).

\section{References}

[1] G. Nie and S. Zhang and P. Liu, The entire logistics integration platform architecture analysis, Journal of logistics Technology, vol. 28, pp. 137-141, 2009.

[2] J. Wei, Electronic commerce logistics management, Chongqing: Chongqing University press, pp. 208-209, 2008.

[3] A. Asosheh, H. S. Nejad and H. K. Kari, A model of a localized cross-border e-commerce, iBusiness, vol. 4, pp. 136-145, 2012. 
[4] Y. Liu and C. Shi, Study online supply chain finance model,Oriental Culture, vol. 6, pp. 83, 2012.

[5] X. Weng and J, An. study commodity connotation and full e-commerce logistics model, Business Studies, vol. 12, pp. 191-194, 2013.

[6] N. Terzia, The impact of e-commerce on international trade and employment, Procedia Social and Behavior - al Sciences, vol. 24, pp. 745-753, 2011.

[7] H. Wang, Logistics financial service innovation research, Fudan University, 2008

[8] L. Sun, Construction Ningbo international commodity trading center of the whole idea and proposed measures, Port Economy, vol. 11, pp. 33, 2011.

[9] Jiusan Society Committee of Beijing, Focus on cross-border e-commerce development, Beijing Observation, vol. 11, pp. 28-29, 2012.

[10] J. Tang, Commodity problems and countermeasures in the development of electronic commerce , Chinese Transportation Development Peak BBS Special Issue, vol. 10, pp. 80-81, 2014. 